\title{
EARLY DIAGNOSIS OF DEMENTIA BASED ON INTERSUBJECT WHOLE-BRAIN DISSIMILARITIES
}

\author{
S. Klein ${ }^{1 *}$, M. Loog ${ }^{2}$, F. van der Lijn ${ }^{1}$, T. den Heijer ${ }^{3,4}$, A. Hammers ${ }^{5,6}$, M. de Bruijne ${ }^{1,7}$, \\ A. van der Lugt ${ }^{8}$, R.P.W. Duin ${ }^{2}$, M.M.B. Breteler ${ }^{3}$, and W.J. Niessen ${ }^{1,9}$

\begin{abstract}
${ }^{1}$ Biomedical Imaging Group Rotterdam, Departments of Radiology \& Medical Informatics, Erasmus MC, Rotterdam, the Netherlands. ${ }^{2}$ Pattern Recognition Laboratory, Faculty of Electrical Engineering, Mathematics, and Computer Science, Delft University of Technology, the Netherlands, m.loog@ tudelft.nl. ${ }^{3}$ Department of Epidemiology \& Biostatistics, Erasmus MC, Rotterdam, the Netherlands. ${ }^{4}$ Department of Neurology, Erasmus MC, Rotterdam, the Netherlands. ${ }^{5}$ MRC Clinical Sciences Centre, Hammersmith Hospital, and Division of Neuroscience and Mental Health, Imperial College, London, UK. ${ }^{6}$ Neurodis Foundation, CERMEP PET centre, Lyon, France. ${ }^{7}$ Department of Computer Science, University of Copenhagen, Copenhagen, Denmark. ${ }^{8}$ Department of Radiology, Erasmus MC, Rotterdam, the Netherlands. ${ }^{9}$ Imaging Science \& Technology, Department of Applied Sciences, Delft University of Technology, the Netherlands.
\end{abstract}

\begin{abstract}
This article studies the possibility of detecting dementia in an early stage, using nonrigid registration of MR brain scans in combination with dissimilarity-based pattern recognition techniques. Instead of focussing on the shape of a single brain structure, we take into account the shape differences within the entire brain. Imaging data was obtained from a longitudinal, population based study of the elderly. A set of 29 subjects was identified, who were asymptomatic at the time of scanning, but were diagnosed as having dementia within 0.7 to 5 years after the scan, and a set of 29 age and gender matched healthy controls were selected. Each subject was registered to all other subjects, using a nonrigid registration algorithm. Based on statistics of the deformation field in the brain, a dissimilarity measure was calculated between each pair of subjects, yielding a $58 \times 58$ dissimilarity matrix. A $k \mathrm{NN}$ classifier was trained on the dissimilarity matrix and the performance was tested in a leave-one-out experiment. A classification accuracy of $81 \%$ was attained (spec. $83 \%$, sens. $79 \%$ ). This demonstrates the potential of whole-brain intersubject dissimilarities to aid in early diagnosis of dementia.
\end{abstract}

Index Terms-dementia, classification, image registration, dissimilarity, brain imaging

\section{INTRODUCTION}

Early diagnosis of dementia has received increasing attention in light of the potential disease-modifying treatments becoming available [1]. By morphological analysis of MR brain scans, the disease may be detected long before the occurrence of clinical symptoms such as memory loss. The most important example is a small hippocampal volume, which has

\footnotetext{
* Corresponding author: s.klein@erasmusmc.nl.

We acknowledge financial support from the FET programme within the EU FP7, under the SIMBAD project (contract 213250). M. Loog received financial support from the Innovational Research Incentives Scheme of the Netherlands Research Organization (NWO, VENI-Grant 639.021.611).
}

consistently been found to be an early biomarker of dementia [2-4]. Other brain structures whose shapes have been related to dementia are the amygdala [3], the putamen and thalamus [5], and the ventricles [6]. The brain structure under consideration needs to be delineated accurately, in order to be useful as a biomarker for dementia.

In this article we study the possibility of predicting dementia by considering the entire brain's morphology. A classifier is trained to distinguish healthy subjects from subjects in an early, asymptomatic stage of dementia. We focus on a dissimilarity-based pattern recognition approach [7], in which the subjects are characterised by their pairwise relation to other subjects in the training set, instead of specific individual shape/appearance features that are traditionally used in statistical pattern recognition. To measure the dissimilarity of two brain scans, we perform a nonrigid registration, which provides us with a coordinate transformation that spatially relates the two images. The standard deviation of the logarithm of the Jacobian determinant of the transformation, calculated over the entire brain, is subsequently used as a distance measure between the two subjects. The method thus does not rely on segmentations of specific brain structures.

\section{METHODOLOGY \& EXPERIMENTS}

\subsection{Data}

Imaging data was obtained from the Rotterdam Scan Study, a longitudinal MRI study on age-related diseases [8]. In the period '95-' 96,518 elderly subjects of 55 years and older were scanned on a Siemens $1.5 \mathrm{~T}$ scanner, using an inversion recovery $3 \mathrm{D}$ half-Fourier acquisition single-shot turbo spin echo sequence, acquired in sagittal direction. The images were reconstructed to $128 \times 256 \times 256$ with a voxel dimension of $1.25 \times 1.0 \times 1.0 \mathrm{~mm}$, and corrected for inhomogeneities using N3 [9].

For the experiments in this study, one subset of 29 subjects (mean \pm st.dev. age of $80 \pm 6 \mathrm{yr} ; 19$ female) was iden- 
tified who where cognitively intact at the time of scanning, but developed clinical symptoms of dementia within 5 years $(2.3 \pm 1.3)$ after the scan was taken. The procedure for dementia screening is explained in [3]. A second subset was composed of 29 age/gender matched controls (mean \pm st.dev. age of $80 \pm 6 \mathrm{yr} ; 19$ female), who remained cognitively intact until the last dementia screening in 2005 (or died before). The Mini Mental State Examination (MMSE) scores for both groups were comparable at the time of scanning (demented: mean 25.7, range 20-30; controls: mean 27.4, range 19-30).

\subsection{Image registration based dissimilarity measure}

To measure the dissimilarity of two brain scans, we propose a method based on nonrigid image registration. Given a pair of images $F(\boldsymbol{x})$ and $M(\boldsymbol{x})$, image registration is the task of finding a coordinate transformation $\boldsymbol{T}(\boldsymbol{x})$ that spatially aligns the two images, such that the deformed "moving" image $M(\boldsymbol{T}(\boldsymbol{x}))$ looks similar to the "fixed" image $F(\boldsymbol{x})$. Based on statistics of $\boldsymbol{T}(\boldsymbol{x})$ over the brain region, we can define a measure of the 'anatomical dissimilarity' between $F$ and $M$. The Jacobian determinant of the transformation $J(\boldsymbol{x})=\operatorname{det}[\partial \boldsymbol{T}(\boldsymbol{x}) / \partial \boldsymbol{x}]$ is a measure of volumetric expansion and compression [10]. A value of 1 indicates volume preservation. Values between 0 and 1 indicate local compression and values larger than 1 indicate local expansion. Often, the logarithm of $J$ is used in statistical analyses, to account for the fact that volumetric expansion by a factor of $r$ should be comparable to a volumetric compression of $1 / r$. If two images have a similar anatomy, we may expect $\log (J)$ to be nearly constant over the image domain. Dissimilar anatomies will be characterised by large fluctuations of $\log (J)$ over the image domain. We argue thus that the standard deviation of $\log (J)$, calculated over the entire brain region, reflects anatomical dissimilarity.

For our experiment, all $N=58$ subjects were registered to each other, resulting in a $N \times N$ 'matrix' of coordinate transformations $\left[\boldsymbol{T}_{i j}\right]$, with $i=1, \ldots, N$ and $j=1, \ldots, N$ the fixed and moving image subject number, respectively. The diagonal of this matrix is filled with identity transformations. A dissimilarity matrix $\boldsymbol{D} \equiv\left[D_{i j}\right]$ was constructed by computing the Jacobian based dissimilarity measure for each pair $(i, j)$, and symmetrising:

$$
D_{i j}=\frac{1}{2}\left(\operatorname{stdev} \log \left(J_{i j}\right)+\operatorname{stdev} \log \left(J_{j i}\right)\right)
$$

The brain region in each scan (over which the standard deviation is computed) was extracted by a multi-atlas segmentationby-registration method, using the atlas described in [11]. The registrations were performed using the open source software package elastix [12]. A two-stage approach was opted for, starting with an affine transformation model, followed by nonrigid B-spline based registration. In the affine registration stage, mutual information [13] was used as a cost function, in combination with a stochastic gradient descent optimisation method with adaptive step size estimation [14]. In the second stage, a B-spline transformation model [15] was employed, embedded in a four-level multiresolution framework, with isotropic control point spacings of $60,60,30$, and $15 \mathrm{~mm}$ in the subsequent resolution levels. Localised mutual information was used as a cost function, integrated in a stochastic optimisation procedure as described in [16]. Exact registration parameter settings can be found in the elastix parameter file database ${ }^{1}$.

\subsection{Dissimilarity-based classification}

Matrix $\boldsymbol{D}$ forms the basis of a classifier that distinguishes between healthy subjects and subjects in an early stage of dementia. Row (or column) $i$ of the dissimilarity matrix $\boldsymbol{D}$ implicitly characterises subject $i$. The subject is thus described by its anatomical dissimilarity with respect to all other subjects. Following the paradigm of [7], the rows of $D$ may be treated as feature vectors, and used in a traditional statistical pattern recognition framework, such as linear discriminant analysis (LDA), logistic regression (LR), or $k$-nearestneighbour $(k \mathrm{NN})$ classification. In this study, we implemented a $k \mathrm{NN}$ classifier (with $k$ estimated automatically from the training set by leave-one-out cross-validation).

Note that this approach is conceptually different from the multidimensional scaling (MDS) [7] techniques, as used in [17] for example, in which feature vectors of a user-defined dimension $M$ are estimated such that their pairwise Euclidean distances approximate $D_{i j}$. Another often used approach is to use the dissimilarities $D_{i j}$ directly as the distances in a $k \mathrm{NN}$ classification framework, as if $\boldsymbol{D}$ were a matrix of pairwise Euclidean distances ${ }^{2}$. This, however, implies that possible correlations between the distances to different subjects are not fully exploited. Treating the rows of $\boldsymbol{D}$ as feature vectors, as advocated in [7], ensures that correlations between rows are taken into account. Another advantage of this technique is that the measure of dissimilarity is neither assumed to be Euclidean nor metric.

The classification experiments were performed in a leaveone-out fashion, using the Matlab ${ }^{\circledR}$ toolbox PRTools ${ }^{3}$.

\section{RESULTS}

The dissimilarity matrix is visualised in Figure 1 . The bottom graph presents the classification result of the dissimilarity based classifier. In total, $81 \%$ of the subjects were correctly classified (spec. $83 \%$, sens. $79 \%$ ).

For comparison, we trained additional classifiers on the left and right hippocampus volumes (normalised by intracranial volume), and on the MMSE score. The hippocampus volume was automatically segmented by the method described in [18]. In a leave-one-out setting, the highest classification

\footnotetext{
${ }^{1}$ http://elastix.isi.uu.nl/wiki.php

${ }^{2}$ In fact, this is equivalent to MDS with $M=N-1$.

${ }^{3}$ http://www.prtools.org
} 


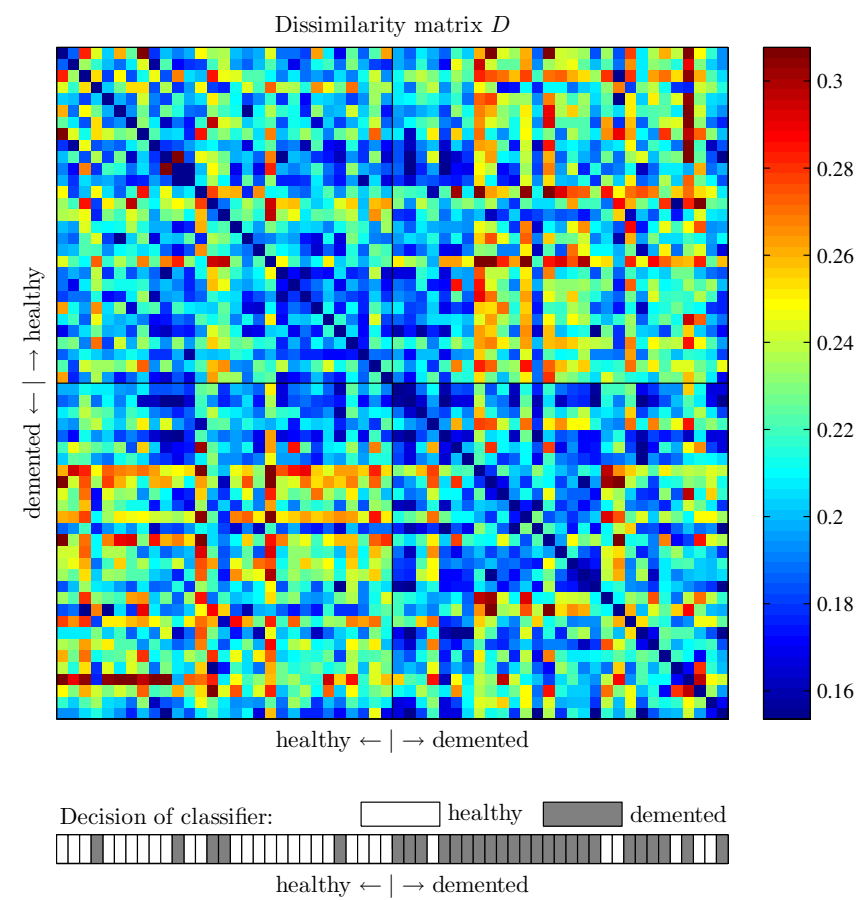

Fig. 1. The dissimilarity matrix $\boldsymbol{D}$ (colour scale chosen to maximise contrast) and the classifier's decision. The dissimilarity matrix roughly has a block structure, corresponding to the groups; elements in the off-diagonal blocks tend to have larger values than those in the diagonal blocks.

performance of $78 \%$ was obtained for the right hippocampus volume (spec. $72 \%$, sens. 83\%). The left hippocampus volume resulted in an accuracy of $74 \%$ (spec. $72 \%$, sens. $76 \%$ ). The MMSE score yielded a prediction accuracy of 66\% (spec. $76 \%$, sens. $55 \%)$.

\section{DISCUSSION}

The results suggest that, within our selected study population, whole-brain dissimilarity-based classification has a slightly larger predictive value for dementia than the hippocampus volume. The whole-brain approach has the advantage that accurate segmentation of brain structures and prior knowledge on which structures are the most relevant are not necessary.

Previous work on MR image based detection of dementia, or more specifically Alzheimer's disease (AD), has mainly focussed on the hippocampal shape. The work by Klöppel et al. [19] is most related to ours. They segment the MR brain images into grey matter, white matter, and cerebro-spinal fluid. After registration to a population template, the (whole-brain) grey matter segmentations are used as feature vectors in a support vector machine (SVM). The method was tested on three groups. The patients in all groups were clinically diagnosed as demented at the time of scanning. On Group I and II, correct-classification rates of $95 \%$ and $93 \%$ were achieved, but the patients in these groups suffered from rather severe, 'definite' AD at the time of scanning. Group III is most comparable to our population, since it only contained patients with 'probable mild' AD. In this group, consisting of 57 controls and 33 patients, $81 \%$ of the subjects were correctly classified (spec. 93\%, sens. 61\%). An improved accuracy of $86 \%$ was obtained by taking into account only a volume of interest (antero-medial lobe) centred around the hippocampus.

Duchesne et al. [20] also employ a population template to which all subjects are aligned by nonrigid registration. An SVM classifier is constructed, using feature vectors formed by the normalised image intensities and Jacobian determinant values within a volume of interest centred on the medial temporal lobes. Leave-one-out validation on a group of 75 subjects suffering from 'mild to moderate probable' $\mathrm{AD}$ and 75 normal controls resulted in an accuracy of $92 \%$.

Miller et al. [17] consider (automatically) segmented hippocampi, which are registered to each other in order to construct a dissimilarity matrix based on the deformation metric implied by their diffeomorphic registration approach. MDS followed by LDA was used for classification. The method was validated on 56 subjects, of whom 36 were healthy and 20 had 'very mild' or 'mild' dementia. With optimal settings, $77 \%$ of the subjects were correctly classified.

Ferrarini et al. [6] focus on the ventricular shape. Based on a shape model, surface nodes significantly related to the presence of $\mathrm{AD}$ are selected. These biomarker nodes are used by a SVM to classify the subjects. A leave-one-out test on a set of 28 healthy controls and 29 patients with probable AD resulted in a classification accuracy of $84 \%$. The experiment involved a training/tuning stage on the full set though. Running the method with the same settings on 29 other patients with probable AD resulted in a sensitivity of $76 \%$.

In summary, our results are in line with those reported in the literature, taking into account that all our subjects were cognitively intact at the time of scanning. The large variety of brain structures that were reported in the literature to be related to dementia was the motivation for our whole-brain approach. The results of [19] do suggest though that it could be beneficial to additionally take into account some volume of interest around the hippocampus. We plan to investigate this aspect in future work, by testing the method for different volumes of interest.

In the proposed method, each subject is characterised by its dissimilarities to multiple subjects in the training set, rather than using a single template as for example in $[19,20]$. This results in a much richer representation of each subject and, in addition, makes the method potentially more robust for misregistrations, just as multi-atlas segmentation-by-registration methods tend to outperform single-atlas based segmentation methods [21]. We may expect that there is an optimum composition of the training set, which includes 'typical examples' (prototypes) of healthy and demented subjects. Determining these by means of a feature selection algorithm forms part of our future work. 


\section{CONCLUSION}

A dissimilarity-based classification framework was proposed that, based on the presence of structural abnormalities in the entire brain, aims to recognise subjects that will develop clinical symptoms of dementia in the near future. The method was tested in a leave-one-out experiment on 58 subjects. All subjects were asymptomatic at the time the MR brain scans were acquired. For 29 subjects, clinical diagnosis of dementia was established 0.7 to 5 years later. The method correctly classified $81 \%$ of all 58 subjects, demonstrating the potential of whole-brain intersubject dissimilarities to aid in early diagnosis of dementia. We would like to emphasise the unique nature of our dataset among those described in the literature; clearly it should be much harder to predict conversion over five years than to diagnose even mild AD.

More generally, the proposed combination of dissimilaritybased classification with nonrigid image registration may prove effective in other applications as well, since it does not rely on a dedicated set of features characterising the disease of interest.

\section{REFERENCES}

[1] E. Scarpini, P. Scheltens, and H. Feldman, "Treatment of Alzheimer's disease: current status and new perspectives," Lancet Neurology, vol. 2, no. 9, pp. 539547, 2009.

[2] P. Scheltens, N. Fox, F. Barkhof, and C. De Carli, "Structural magnetic resonance imaging in the practical assessment of dementia: beyond exclusion," Lancet Neurology, vol. 1, no. 1, pp. 13-21, 2002.

[3] T. den Heijer, M.I. Geerlings, F.E. Hoebeek, A. Hofman, P.J. Koudstaal, and M.M.B. Breteler, "Use of hippocampal and amygdalar volumes on magnetic resonance imaging to predict dementia in cognitively intact elderly people.," Arch. Gen. Psychiatry, vol. 63, no. 1, pp. 57-62, 2006.

[4] D.P. Devanand, G. Pradhaban, X. Liu, A. Khandji, S. De Santi, S. Segal, H. Rusinek, G.H. Pelton, L.S. Honig, R. Mayeux, Y. Stern, M.H. Tabert, and M.J. de Leon, "Hippocampal and entorhinal atrophy in mild cognitive impairment: prediction of Alzheimer disease.," Neurology, vol. 68, no. 11, pp. 828-836, 2007.

[5] L.W. de Jong, K. van der Hiele, I.M. Veer, J.J. Houwing, R.G.J. Westendorp, E.L.E.M. Bollen, P.W. de Bruin, H.A.M. Middelkoop, M.A. van Buchem, and J. van der Grond, "Strongly reduced volumes of putamen and thalamus in Alzheimer's disease: an MRI study," Brain, vol. 131, pp. 3277-3285, 2008.

[6] L. Ferrarini, W.M. Palm, H. Olofsen, R. van der Landen, M.A. van Buchem, J.H.C. Reiber, and F. Admiraal-Behloul, "Ventricular shape biomarkers for Alzheimer's disease in clinical MR images," Magnetic Resonance in Medicine, vol. 59, pp. 260-267, 2008.

[7] E. Pȩkalska and R.P.W. Duin, The Dissimilarity Representation for Pattern Recognition, Foundations and Applications, World Scientific, Singapore, 2005.

[8] T. den Heijer, S.E. Vermeer, R. Clarke, M. Oudkerk, P.J. Koudstaal, A. Hofman, and M.B. Breteler, "Homocysteine and brain atrophy on MRI of non-demented elderly.," Brain, vol. 126, no. Pt 1, pp. 170-175, 2003.

[9] J.G. Sled, A.P. Zijdenbos, and A.C. Evans, "A nonparametric method for automatic correction of intensity nonuniformity in MRI data.," IEEE Transactions on Medical Imaging, vol. 17, no. 1, pp. 87-97, 1998.

[10] T. Rohlfing, C.R. Maurer Jr., D.A Bluemke, and M.A. Jacobs, "Volume-preserving nonrigid registration of MR breast images using free-form deformation with an incompressibility constraint," IEEE Transactions on Medical Imaging, vol. 22, no. 6, pp. 730-741, 2003.

[11] A. Hammers, R. Allom, M.J. Koepp, S.L. Free, R. Myers, L. Lemieux, T.N. Mitchell, D.J. Brooks, and J.S. Duncan, "Three-dimensional maximum probability atlas of the human brain, with particular reference to the temporal lobe," Human Brain Mapping, vol. 19, no. 4, pp. 224-247, 2003.

[12] S. Klein, M. Staring, K. Murphy, M.A. Viergever, and J.P.W. Pluim, "elastix: a toolbox for intensity-based medical image registration," IEEE Transactions on Medical Imaging, vol. 29, no. 1, pp. 196-205, 2010.

[13] P. Thévenaz and M. Unser, "Optimization of mutual information for multiresolution image registration," IEEE Transactions on Image Processing, vol. 9, no. 12, pp. 2083-2099, 2000.

[14] S. Klein, J.P.W. Pluim, M. Staring, and M.A. Viergever, “Adaptive stochastic gradient descent optimisation for image registration," International Journal of Computer Vision, vol. 81, no. 3, pp. 227-239, 2009.

[15] D. Rueckert, L.I. Sonoda, C. Hayes, D.L.G. Hill, M.O. Leach, and D.J. Hawkes, "Nonrigid registration using free-form deformations: Application to breast MR images," IEEE Transactions on Medical Imaging, vol. 18, no. 8, pp. 712-721, 1999.

[16] S. Klein, U.A. van der Heide, I.M. Lips, M. van Vulpen, M. Staring, and J.P.W. Pluim, "Automatic segmentation of the prostate in 3D MR images by atlas matching using localized mutual information," Medical Physics, vol. 35, no. 4, pp. 1407-1417, 2008.

[17] M.I. Miller, C.E. Priebe, A. Qiu, B. Fischl, A. Kolasny, T. Brown, Y. Park, T. Ratnanather, E. Busa, J. Jovicich, P. Yu, B.C. Dickerson, R.L. Buckner, and the Morphometry BIRN, "Collaborative computational anatomy - an MRI morphometry study of the human brain via diffeomorphic metric mapping," Human Brain Mapping, vol. 30, no. 7, pp. 2132-2141, 2009.

[18] F. van der Lijn, T. den Heijer, M.M.B. Breteler, and W.J. Niessen, "Hippocampus segmentation in MR images using atlas registration, voxel classification, and graph cuts," $\mathrm{Neu}$ rolmage, vol. 43, no. 4, pp. 708-720, 2008.

[19] S. Klöppel, C.M. Stonnington, C. Chu, B. Draganski, R.I. Scahill, J.D. Rohrer, N.C. Fox, C.R. Jack Jr., J.A. Ashburner, and R.S.J. Frackowiak, "Automatic classification of MR scans in Alzheimer's disease," Brain, vol. 131, no. 3, pp. 681-689, 2008.

[20] S. Duchesne, A. Caroli, C. Geroldi, C. Barillot, G.B. Frisoni, and D.L. Collins, "MRI-based automated computer classification of probable AD versus normal controls," IEEE Transactions on Medical Imaging, vol. 27, no. 4, pp. 509-520, 2008.

[21] T. Rohlfing, R. Brandt, R. Menzel, and C.R. Maurer Jr., "Evaluation of atlas selection strategies for atlas-based image segmentation with application to confocal microscopy images of bee brains," NeuroImage, vol. 21, no. 4, pp. 1428-1442, 2004. 